beiden Glovertürme des Innen- und AuBenringes mit ihrer verstärkten Nitrosezufuhr wird die Hauptreaktion von den Kammern in die vor diesen geschalteten Türme verlegt." Er spricht also von beiden Türmen, und unmittelbar darauf sagt er :, „,Der Doppelring hat auch da seinen Platz, wo man möglichst wenig unreine Säure durch Produktion im Glover gewinnen will, denn er ermöglicht, fast die gesamte Menge der salpetrigen Säure in $54-58^{\circ}$ iger Schwefelsäure zu absorbieren und diese Nitrose im 2. Glover, in den Flugstaub nicht mehr gelangt, zu verarbeiten, während der erste Glover nur die aus den letzten Spuren salpetriger Säure gebildete $60^{\circ}$ ige Nitrose erhält. Hierdurch wird die Säureproduktion im ersten Glover, der schmutzige Säure liefert, auf ein geringes MaB beschränkt." Im ersten Satz schreibt er beiden Türmen große Säureproduktion zu, und im zweiten hebt or die geringe Säureproduktion des Glovers 1 als einen Vorteil seines Verfahrens hervor. P e t e r s e n s Arbeitsweise hat Ahnlichkeit mit der nach dem Patent 140 825. Daß man aber nach seinem Verfahren besser arbeitet, will mir nicht einleuchten. P e t e r s o n sucht allerdings don Beweis dafür zu erbringen, denn er weist in seinem Vortrage darauf hin, daß die Kunigundenhütte in Oberschlesien sein Verfahren in jüngster Zeit in Betrieb genommen habe. Er sagt darüber wörtlich : „Das System, welches von einem großen Installationsbureau im vorigen Jahre gebaut und in Betrieb genommen wurde, gemeint kann wohl nur $\mathrm{N}$ i e d e $\mathrm{n} f$ ü $\mathrm{h} r$ sein, hatte die geteilte Gloverfunktion $2500 \mathrm{cbm}$ Kammerraum und zwei GayLussactürme. Das System erzielte, nachdem ein regelmäBiger Betrieb herbeigeführt war, im dreimonatlichen Durchschnitt eine tägliche Produktion von $12 \mathrm{t} 50^{\circ}$ Säure, gleich $4,8 \mathrm{~kg}$ auf $1 \mathrm{cbm}$ Kammerraum bei einem Verbrauch von 2,7\% Salpetersäare von $38^{\circ}$ Bé. Die geteilte Gloverfunktion wurde aber dann aufgegeben, die Einrichtungen nach $\mathrm{P}$ e t e r s e n getroffen, und nun stieg die Produktion auf $9 \mathrm{~kg}$ pro $1 \mathrm{cbm}$, wobei der Salpetersäureverbrauch auf $1,1 \%$ sank.

Wenn nun P e t e r s e n glaubt, daß der große Salpetersäureverbrauch von $2,7 \%$ bei nur $4,8 \mathrm{~kg}$ Säureproduktion auf $\mathbf{l} \mathrm{cbm}$ in der $\mathrm{N}$ i e d e $\mathbf{n} \mathrm{f} \ddot{\mathrm{u}} \mathrm{h} \mathbf{r}$ schen Arbeitsweise seinen Grund hat, so will ich ihm darauf erwidern, da $\beta$ ich bei $7 \mathrm{~kg}$ pro $1 \mathrm{cbm}$ heute nur ein Fünftel der Salpetersäure verbrauche, die er angibt, allerdings beziehen sich seine Angaben auf Zinkblende.

M. H.! Ich habe nicht etwa den Beweis erbringen wollen, da $B$ man mit zwei Glovern auf alle Fälle rationeller arbeite als mit einem. Ich wollte nur die Behauptung widerlegen, daß durch die Teilung der Gloverfunktionen diese unbedingt eine Einbuße erleiden müßten.

\section{Über die Frage der Feuergefährlichkeit von Kunstwollen.}

\section{Von W. H. Schramm, Bielitz.}

(Eingeg. den 3./1. 1908.)

Von einer großen Schiffahrtsgesellschaft werden Kunstwollen gegenwärtig nur als Deckfracht angenommen, da sie wegen ihres Gehaltes an Fett- stoffen und pflanzlichen Fasern angeblich der Neigung zur Selbstentzündung verdächtig und deshalb feuergefährlich seien.

Da hingegen andere Schiffahrtsgesellschaften Kunstwollen im Innern der Schiffe verfrachten, und auch langjährige Erfahrungen der Kunstwollfabriken gegen eine erhebliche Feuergefährlichkeit der Kunstwollen sprechen, wurde von einer großen Speditionsfirma an die Versuchsstation der K. K. Staatsgewerbeschule in Bielitz das Ansuchen gestellt, ein Gutachten über die Frage der Feuergefährlichkeit von Kunstwollen abzugeben.

Es war von vornherein klar, daß jede Feststellung, die zur Beantwortung dieser Frage führen sollte, nur im Vergleichen der Kunstwolle mit anderen ähnlichen Stoffen, demnach nur verhältnismäBig geschehen konnte. Die zu diesem Zwecke nächstliegenden Stoffe sind rohe Baumwolle, rohe, ungewaschene Schafwolle, sog. Schweißwolle und wohl auch gewaschene Schafwolle. Alle diese werden nicht nur im Innern der Schiffe verfrachtet, sondern es ist auch ihr Verhaiten beim Lagern und beim Transport bekannt.

Uber die Selbstentz ü.ndung von Faserstoffen ist freilich nur wenig bekannt geworden, doch kann das Folgende wohl als festgestellt erachtet werden 1 ) :

1. Baumwollstückware, die auf einer größeren Fläche durch und durch von Leinöl durchdrungen wurde, entzündete sich im Sommer beim Lagern in einem trockenen Raume von selbst 2 ).

2. Mit Ollack getränkte Watte verkohlte schon bei gewöhnlicher Temperatur im Innern von selbst und entzündete sich beim Auseinanderfalten ${ }^{3}$ ).

3. Abfälle von Baumwolle, Leinen, Jute und Wolle, die mit fetten Olen (Seehund- oder Walfischtran, Olivenöl, Baumwollsamenöl, Rüböl) g e t r ä $n \mathrm{k}$ t wurden, entzündeten sich nach 3-8 Stunden von selbst oder verkohlten, wenn sie unter Luftabschluß von außen auf $82^{\circ}$ erwärmt wurden ${ }^{4}$ ).

4. Reine Baumwolle, die mit dem Doppelten ihres Gewichtes an fetten Olen getränkt und bei mäßiger Luftzufuhr von außen auf $100^{\circ}$ erhitzt wurde, zeigte im Innern je nach der Art des angewendeten Oles oft schon nach einer Stunde eine heftige Temperatursteigerung, die bis zur Selbstentzündung führen konnte ${ }^{5}$ ).

5. Putzwollen und der ölgetränkte Ausputz aus den Wollkratzen entzünden sich leicht von selbst, wenn sie an warmen Orten und yor Luftzutritt einigermaßen geschützt liegen bleiben.

6. Bei Baumwolle, die mit der gleichen Gewichtsmenge Rüböl oder Baumwollsamenöl getränkt und unter möglichster Beschränkung des Luftzutrittes von außen auf $25^{\circ}$ erwärmt wurde,

1) Altere Angaben sind in der Schrift ,Die Selbstentzündung von Schiffsladungen, Baumwolle und anderen Faserstoffen usw." von Dr. L. H ä $p k$ e gesammelt zu finden. 231.

2) L. S c h r e i n e r, Färber-Ztg. (Lehne) 1897,

3) W. Li p pert, diese Z. 11, 435 (1897).

4) J. J. Colo m a n n, Jahresbericht f. chem. Technologie 1876, 931.

$\left.{ }^{5}\right) \mathrm{M} \mathrm{a} \mathrm{ckey}$, J. Soc. Chem. Ind. 1896, 91; nach B en edikt U l z er, Analyse der Fette, 1903,438 . 
war die durch die Autooxydation bedingte Wärmeerzeugung so gering, daß die Gefahr einer durch diesen Vorgang hervorgerufenen Selbstentzündung sehr klein erschien. Selbst bei Anwendung von Leinöl wurde die Gefahr noch als ziemlich fernliegend, bei Leinölfirnis dagegen als sehr naheliegend erachtet ${ }^{6}$ ).

7. Unter den riesigen Wollmassen, die in den Londoner Docks lagern und die weite Reise von Australien und vom Kap gemacht haben, sind nie Selbstentzündungen beobachtet worden, obwohl sie in großen und schweren Ballen zusammengepreßt liegen und ungewaschen, $d$. i. fetthaltig sind. Allerdings sind Temperaturen von $80-90^{\circ}$ im Innern dieser Ballen beobachtet worden, doch $\mathrm{zu}$ einer Verkohlung kam es nie ${ }^{7}$ ).

8. Hingegen scheint die Meinung herrschend zu sein, daß rohe Baumwolle zur Selbstentzündung neige ${ }^{8}$ ).

Aus dem Angeführten erklärt es sich, wie die vielfach verbreitete Meinung von einer erheblichen Feuergefährlichkeit der Kunstwollen entstehen konnte. Die Kunstwollen schienen einerseits durch ihren Gehalt an Baumwolle oder anderen pflanzlichen Fasern, andererseits durch ihren Gehalt an Spick- oder Schmälzöl verdächtig. Ưber die Höhe des Gehaltes an Fettstoffen scheinen allerdings vielfach übertriebene Vorstellungen zu bestehen. Wie weit hierzu die im Handbuch der Färberei von Loewenthal, Knecht und Rawson, 2. Aufl, 259, enthaltene Angabe, daß aus Kunstwolle gesponnene Garne zuweilen mehr als $15 \%$ ihres eigenen Gewichtes an 01 enthalten, beigetragen hat, möge dahingestellt sein. Ein so hoher Fettgehalt mag bei aus sehr schlechtem Material gesponnenen Garnen ja mitunter notwendig sein, bei unversponnenen Kunstwollen werden die $\mathrm{Er}$ zeuger derselben schon in ihrem eigenen Interesse den Zusatz von Spickmitteln prozentuell weit niedriger halten. Eine Kunstwollfabrik in Biala z. B. pflegt nach ihrer Angabe ihre Erzeugnisse nur mit $5 \%$ Elain einzufetten, was durch die Analyse dieser Kunstwollen sich auch bestätigte.

Um nun zunächst einmal eine Grundlage zur Behandlung der ganzen Frage zu schaffen, wurde bei dreien der von der bereits genannten Kunstwollfabrik zur Verfügung gestellten Kunstwollsorten der Gehalt an pflanzlicher Faser und Fett bestimmt.

Die Analyse lieferte folgende Ergebnisse:

Kunst- Kunst. Kunstwolle I wolle II wolle III

$\% \quad \%$

Wasser . . . . . . . . . . $10,00 \quad 10,32 \quad 9,95$

Petrolätherlösliches (Fettstff.) $5,12 \quad \mathbf{1 , 0 0} 5,02$

Pflanzliche Faser . . . . . 2,30 $8,10 \quad 2,46$

Zieht man zum Vergleich die rohe Schafwolle heran, so ergibt sich, daß diese mit einem Fettgehalt von etwa $6-21 \%$ den Fettgehalt der untersuchten

$\left.{ }^{6}\right)$ R. K i B ling, diese Z. 8, 44 (1895).

7) H. S i l b e r m a n n, Färber-Ztg. (Lehne) $1900,187$.

8) Gegen die Gültigkeit dieser Meinung würden die Versuche K i B 1 i n g s a. a. O. sprechen, der bei Wolle eine größere Wärmeentwicklung mit Leinölfirnis fand, als bei Baumwolle.
Kunstwollen weit überragen könne. Die gewaschenen Wollen sollen zweckmäßig noch einen Fettgehalt von $2,5-3,5 \%$ aufweisen, Kammgarne enthalten $2-3 \%$ Ol. Rohe Baumwolle soll hingegen nicht mehr als $0,4-0,5 \%$ ihres Gewichtes an Petroläther abgeben.

Ebenso enthalten die überseeischen Wollen meist einen größeren Gehalt von pflanzlichen Stoffen (Kletten und andere Pflanzenteile) als die untersuchten Kunstwollen.

Trotzdem kann aus den angeführten Tatsachen, daß ungewaschene Wollen meist weit mehr, gewaschene Wollen meist bedeutend weniger Fettstoffe enthalten, als die untersuchten Kunstwollen, und die letzteren sich auch ärmer an pflanzlichen Stoffen erwiesen als viele der überseeischen rohen Schafwollen, nicht ohne weiteres der Schluß gezogen werden, daß Kunstwollen mindestens nicht feuergefährlicher sein können, als rohe Schafwollen.

Gegen eine solche Schlußfolgerung können nämlich folgende durchaus berechtigte Einwände erhoben werden :

1. Die Wärmeentwicklung, die durch irgend ein auf Faserstoffen verteiltes $O \mathrm{l}$ oder Fett bewirkt wird, hängt von der Oxydationsfähigkeit des betreffenden Oles ab. Nun besitzt das natürliche Wollfett, das zum größten Teile aus Estern höherer Fettalkohole besteht, gewiß eine geringere Oxydationsfähigkeit, als die gebräuchlichen Wollspickmittel. Die Höhe der Jodzahl eines Oles kann ein gewisses $\mathrm{Maß}$ abgeben für die Größe seiner Oxydationsfähigkeit.

Da besitzt nun das

$$
\text { Jie }
$$$$
\text { Wollfett . . . . . . . . . . } 20-21
$$$$
\text { Elain . . . . . . . . . . . . } 80
$$$$
\text { Olivenöl . . . . . . . . . . . } 82,8
$$$$
\text { Rüböl . . . . . . . . . . . . . } 101
$$$$
\text { Baumwollsamenöl . . . . . . . . } 108
$$$$
\text { Leinöl } l^{9} \text { ) . . . . . . . . . . . . . . . } 170
$$

2. Die Wärmeentwicklung hängt auch von der Feinheit der Faserstoffe ab. Gewiß sind aber Kunstwollen, die bereits so viele chemische und mechanische Operationen durchzumachen hatten, in diesem Sinne ,feiner" geworden als die rohen Schafwollen.

3. Die pflanzliche Faser befindet sich in den Kunstwollen ebenfalls in feinerer und gleichmäBigerer Verteilung als bei den rohen Schafwollen.

$\mathrm{Da}$ es mit den bisherigen Erfahrungen also nicht möglich war, zu einem sicheren Urteil über die Frage der verhältnismäBigen Feuergefährlichkeit der Kunstwollen zu gelangen, blieb nur übrig, neue Erfahrungen zu sammeln. Dies erschien nur mittels vergleichender Selbstentzündungsversuche möglich.

Solche Versuche sind mit verschiedenen Faserstoffen und mit verschiedenen Olen bereits ausgeführt worden, z. B. von J. J. Cole man n, R. Ki B ling, M a ckey, Lippert u. a.

Die von diesen Autoren angewendeten Methoden konnten indessen für den vorliegenden Fall nicht ohne weiteres angewendet werden, da sie alle

9) Leinöl und Leinölfirnis kommen natürlich für das Einfetten von Wolle nicht in Betracht. 
mit einem Verhältnis von Fett zu Faserstoff arbeiten, das viel zu weit von dem Verhältnis abweicht, das bei den in Betracht kommenden Textilfasern gewöhnlich besteht.

Daß eine Kunstwolle, die mit der gleichen ${ }^{10}$ ) oder der doppelten ${ }^{11}$ ) Gewichtsmenge eines Fettes beladen wurde, sich einer ebenso behandelten Schafwolle oder Baumwolle sehr ähnlich verhalten würde, war ebenso sicher vorauszusehen, wie wertlos für den vorliegenden Fall.

Für diesen erschien es notwendig, die Empfindlichkeit der angewendeten Methode so weit zu erhöhen, daß bei einem Fettgehalt der Faserstoffe, der dem in der Praxis in Betracht kommenden sich doch einigermaßen nähert, bereits eine beträchtliche Wärmeentwicklung auftritt, und auch geringe Unterschiede im Fettgehalt sioh durch eine deutliche Anderung dieser Wärmeentwicklung bemerkbar machen.

Es gelang dies nach einigen vergeblichen Versuchen ${ }^{12}$ ) auf eine sehr einfache Weise durch Erhitzung der Faserstoffe in einem der gebräuchlichen Konditionierapparate auf $110^{\circ}$.

Das zu prüfende Fasermaterial wurde meist in der Menge von $50 \mathrm{~g}$, mitunter aber auch in größerer Menge in ein beiläufig kugelförmiges Körbchen aus verzinntem Eisendraht von $10 \mathrm{~cm}$ Höhe und Breite ziemlich fest eingepackt, so daß es ein in die Mitte eingestecktes Thermometer an dessen unterem Teile innig umbüllte. Das Körbchen wurde dann in den Konditionierapparat gebracht, wobei der obere Teil des in dem Körbchen steckenden Thermometers durch die mittlere kleine Offnung. im Deckel des Konditionierapparates aus diesem herausragte. Die Temperatur wurde vor dem Einbringen des Körbsohen im Konditionierapparat auf $110^{\circ}$ eingestellt und, soweit es möglich war, während des ganzen Versuches konstant erhalten. Zur Erhöhung der Sicherheit wurde noch ein drittes Thermometer neben dem Körbchen angebracht. Nun wurden von Zeit zu Zeit Temperaturablesungen vorgenommen, und zwar so lange, bis der Unterschied in den. Ablesungen an den beiden Thermometern, die die Temperatur im Konditionierapparate angaben, und an dem Thermometer, das die Temperatur innerhalb des Fasermaterials zeigt, einen deutlichen $\mathrm{Ab}$ fall oder eine lange andauernde Gleichmäßigkeit aufwies.

Zur näheren Erläuterung des angewendeten Verfahrens und seiner Empfindlichkeit sollen folgende Versuche mit Baumwolle angeführt werden.

$$
\begin{gathered}
\text { Selbstentzündungsversuche mit } \\
\text { Baumwolle. }
\end{gathered}
$$

Rohe Baumwolle mit einem ursprünglichen, natürlichen Fettgehalte von $0,17 \%$ wurde mit wechselnden Mengen Elain möglichst gleichmäBig getränkt. Die entsprechende Elainmenge wurde in Petroläther gelöst auf die Baumwolle gebracht, diese dann tüchtig durchgeknetet und ausgebreitet so lange an die Luft gelegt, bis der Petroläther freiwillig abgedunstet war.

10) Nach K i B I i n g.

11) Nach Mackey.

12) Z. B. auch mit dem von $M$ a e $k$ e $y$ angegebenen Apparat.
Das Elain, war das von der genannten Kunstwollfabrik zum Spicken von Kunstwolle ausschließ. lich verwendete Elain, ein normales Handelselain, das eine normale Jodzahl von 81 und einen geringen Gehalt an Unverseifbarem und Wasser aufwies.

Mit 6\% ihres Gewichtes Elain getränkte Baum. wolle ergab im Innern eine Temperaturerhöhung bis zu $13^{\circ}$. Das Maximum war nach $21 / 2$ Stunden erreicht.

Mit 10\% Elain getränkte Baumwolle ergab nach $21 / 2$ Stunden ein Maximum von $17^{\circ}$. Nach dem Auseinanderfalzen zeigte sie sich im Innern durch oxydierte Ölsäure deutlich gelblicher gefärbt.

Mit 15\% Elain getränkte Baumwolle ergab nach $21 / 2$ Stunden ein Maximum von $37^{\circ}$. Nach dem Auseinanderfalten zeigte sie sich im Innern etwas gebräunt.

Mit $\mathbf{2 0} \%$ Elain getränkte Baumwolle ergab nach 2 Stunden ein Maximum von $48^{\circ}$. Nach dem Auseinanderfalten zeigte sie sich im Innern deutlich gebräunt, doch trat bei dem Aufblasen von Luft noch kein Glimmen ein.

Mit 30\% Elain getränkte Baumwolle ergab nach $11 / 2$ Stunden ein rasches Ansteigen der Temperatur im Innern um $126^{\circ}$, also von 110 auf $236^{\circ}$. Nach dem Auseinanderfalten zeigte sie im Innern verkohlte, dunkelbraune Stellen, die beim Aufblasen von Luft zu glimmen begannen. Nach einiger Zeit fingen die glimmenden Teile unter Flammenentwicklung zu brennen an.

Mit $60 \%$ Elain getränkte Baumwolle zeigte ein ähnliches Verhalten wie die mit 30\% Elain getränkte, doch war die erstere auch außen verkohlt, während die letztere trotz der Verkohlung im Inneren aussen völlig unverändert geblieben war.

Uberraschenderweise ergab auch die rohe, gar nicht eingefettete Baumwolle eine Temperatur. erhöhung im Innern, und zwar nach 2 Stunden ein Maximum von $9^{\circ}$.

Reine, sog. medizinische Watte, die aber, wie sich später herausstellte $0,39 \%$ Petrolätherlösliches enthielt, ergab nach $21 / 2$ Stunden ein Maximum von $8^{\circ}$.

Rohe Baumwolle, die dreimal je 3 Stunden lang mit Petroläther ausgekocht worden war13), ergab nach $2^{1 / 2}$ Stunden ein Maximum von $9^{\circ 4}$ ).

Aus den angeführten Versuchen geht die für den vorliegenden Fall wichtige Tatsache hervor, daß Baumwolle, dio mit 6-10\% Elain getränkt wurde, sich unter äußeren Bedingungen, die einer Selbstentzündung überaus förderlich sind, fast genau so verhält, wie rohe Baumwolle mit unternormalem Fettgehalt, ja sogar wie möglichist entfetteteBaum. wolle.

13) Nach einer Privatmitteilung des Herrn Dr. Z e ll n e r in Bielitz erreicht man bei pflanzlichen Stoffen auf diese Art viel leichter eine völlige Ent. fettung als bei der kalten Extraktion im Soxh let. schen Apparat.

14) Die Tatsache, daß möglichst vollkommen entfettete Faserstoffe im Innern, wenn sie im Iuftbad längere Zeit erhitzt werden, eine der Temperatur des Luftbades gegenüber erhöhte Temperatur aufweisen, ist gewiß merkwürdig und fordert $\mathrm{zu}$ einer näheren Untersuchung der Ursachen auf. Die Erscheinung an sich ist durch wiederholte Versuche sichergestellt. 
Da außerdem eine länger dauernde von außen kommende Erhitzung von Baumwolle bis auf $110^{\circ}$ beim Transport unter normalen Umständen wohl niemals vorkommen kann, so kann mit Sicherheit ausgesprochen werden, daß eine bis zu $10 \%$ mit Elain gleichmäßig getränkte Baumwolle nicht mehr Anlaß zu Selbstentzündung und Bränden geben wird, als rohe Baumwolle.

Und selbst die mit $15 \%$ Elain beladene Baumwolle kann noch nicht als gefährlich betrachtet werden.

Auf andere Ole wurden die Versuche vorläufig nicht ausgedehnt. Trocknende Öle, die eine viel höhere Oxydationsfähigkeit besitzen als Elain, dürfen in guten Wollspickmitteln nicht enthalten sein, Olivenöl besitzt fast die gleiche Jodzahl wie Elain.

Für das Baumwollsamenöl geht aus den Versuchen $\mathrm{M}$ a c k e y s ${ }^{15}$ ) hervor, daB die Temperaturerhöhung und somit die Neigung zur Selbstentzündung bei Watte, die mit der doppelten Gewichtsmenge Baumwollsamenöl getränkt und unter mäßiger Luftzufuhr von außen auf $100^{\circ}$ erhitzt wurde, ungefähr. um ein Drittel größer ist, als bei dem Parallelversuch mit Elain. Das entspricht auch ungefähr dem Verhältnis der Jodzahlen der beiden Öle. Es ist also wahrscheinlich, daß eine mit 7,5\% Baumwollsamenöl getränkte Baumwolle sich etwa so verhalten wird, wie eine mit $10 \%$ Elain getränkte Baumwolle.

\section{Selbstentzündungsversuche mit $\mathrm{Seh}$ af $\mathrm{folle}$.}

$\mathrm{Nicht}$ gewaschene, sehr unreine ${ }^{16}$ ) rohe Schafwolle, angeblich aus Ungarn stammend, ergab nach 5 Stunden ein Maximum von $12^{\circ}$.

G e w a s c h e n e Wolle, die dreimal durch je 3 Stunden mit Petroläther ausgekocht worden war, ergab nach 3 Stunden ein Maximum von $6^{\circ}$.

Mit $10 \%$ Elain gefettete, gewa$\mathrm{s}$ c h e n e Wolle ergab nach 3 Stunden ein Maximum von $12^{\circ}$.

Hiernach scheint es, als ob Wolle die Oxydation eines oxydablen Öles etwas weniger befördert als Baumwolle.

Nachdem durch die vorstehend 'geschilderten Versuche das Verhalten von mit Elain getränkter Baumwolle und Wolle unter äußeren Bedingungen, die einer Selbstentzündung sehr förderlich sind, studiert worden war, konnte das Verhalten von Kunstwollen unter den gleichen Verhältnissen geprüft werden.

Es geschah dies mit fünf verschjedenen Kunstwollproben, die von seiten der Versuchsstation dem Lagerraum einer Kunstwollfabrik in Biala entnommen wurden.

Strumpfshoddy ergab nach $3^{1 / 2}$ Stunden ein Maximum von $6^{\circ}$.

Droussierte Kunstwolle, rot gefärbt, ergab nach $3^{1 / 2}$ Stunden ein Maximum von $7^{\circ}$.

15) A. a. O.

16) Der Waschverlust wurde mit $64 \%$ bestimmt.
Baumwollhaltige Kunstwolle ergab nach $5^{1 / 2}$ Stunden ein Maximum von $4^{\circ}$.

Auslandzephir ergab überhaupt keine Temperaturerhöhung.

Shoddy aus Decken ergab nach 3 Stunden ein Maximum von $10^{\circ}$.

$$
\text { End ergebnisse. }
$$

1. Mit Elain bis zu $10 \%$ ihres Gewichtes gleichmäßig gefettete rohe, lufttrockene Baumwolle neigt nicht stärker zur Selbstentzündung als, rohe, lufttrockene Baumwolle.

2. Gewaschene, lufttrockene Wolle, die bis zu $10 \%$ ihres Gewichtes gleichmäßig gefettet ist, neigt nicht stärker zur Selbstentsüudung als rohe, ungewaschene Wolle, sog. Schweißwolle.

3. Aus den beiden vorstehenden Sätzen läßt sich bereits mit fast völliger Sicherheit voraussagen, daß irgend eine Kunstwolle mit beliebigem Baumwollgehalt, die nicht mehr als $10 \%$ eines Fettes ent. hält, das eine ähnliche Jodzahl besitzt wie Elain, nicht stärker zur Selbstentzündung neigen wird als ungewaschene Schafwolle oder rohe Baumwolle. Versuche, die mit von seiten der Versuchsstation beliebig ausgewählten Kunstwollproben vorgenommen wurden, bestätigen diese Voraussage vollkom men, ja, sie zeigten bei der hierfür angewandten Prüfungsmethode nicht nur keine größere, sondern teilweise sogar eine geringere Neigung zur Selbstentzündung als rohe ungewaschene Schafwolle oder rohe Baumwolle.

Es könnte noch eingewendet werden, daß bei dem Aufeinanderlagern vieler der ungefähr $2 \mathrm{cbm}$ großen und $200 \mathrm{~kg}$ schweren Kunstwollballen durch den Druck, den die oberen auf die unteren ausüben, eine Art Entmischung, ein Ansammeln des Oles etwa in den untersten Teilen des untersten Ballens eintreten könnte. Hier würde dann der Fall eintreten, daß die Kunstwolle stellenweise weit über $10 \%$ Fett enthielte und infolgedessen stärker zur Selbstentzündung neigen könnte. Eine solche Entmischung ist bei der großen Federkraft der Wollhaare und der innigen Adhäsion zwischen Faser und Öl wohl von vornherein wenig wahrscheinlich. Zur völligen Sicherheit wurde Baumwolle snd gewaschene Schafwolle, die $10 \%$ Elain enthielten, stundenlang einem Druck ausgesetzt, der $50 \mathrm{~kg}$ auf den Quadratdezimeter betrug. Unter die Faserstoffe gelegtes Filter. papier hat aber nur sehr geringe Fettmengen aufgenommen. Ja selbst mit 30\% Elain getränkte Baumwolle, die tagelang dem gleichen Drucke ausgesetzt worden war, gab an das daruntergelegte Filterpapier nur so wenig Fett ab, daß dieses nachher $3 \%$ Fett enthielt.

Auf Grund vorstehender Betrachtungen und Versuche konnte mit voller Sicherheit ausgesprochen werden, daß Kunstwollen keine größere Feuergefährlichkeit besitzen, als rohe ungewaschene Schafwollen oder rohe Baumwollen, daß sie also mit genau derselben Berechtigung im Innern der Schiffe verfrachtet werden können, wie die letztgenannten Faserstoffe.

Bielitz, im Dezember 1907. 